\title{
CEA, CA 15-3, and TPS as Prognostic Factors in the Follow-up Monitoring of Patients After Radical Surgery for Breast Cancer
}

\author{
SARKA SVOBODOVA ${ }^{1}$, RADEK KUCERA ${ }^{1}$, ONDREJ FIALA ${ }^{3,4}$, MARIE KARLIKOVA $^{1}$, \\ ANDREA NARSANSKA ${ }^{2}$, ILONA ZEDNÍKOVÁ ${ }^{2}$, VLADISLAV TRESKA ${ }^{2}$, DAVID SLOUKA ${ }^{1}$, \\ MILENA ROUSAROVA ${ }^{1}$, ONDREJ TOPOLCAN ${ }^{1}$ and JINDRICH FINEK ${ }^{3}$ \\ ${ }^{1}$ Department of Immunochemistry, University Hospital and Faculty of Medicine in Pilsen, \\ Charles University, Pilsen, Czech Republic; \\ ${ }^{2}$ Department of Surgery, University Hospital and Faculty of Medicine in Pilsen, \\ Charles University, Pilsen, Czech Republic; \\ ${ }^{3}$ Department of Oncology and Radiotherapy, University Hospital and Faculty of Medicine in Pilsen, \\ Charles University, Pilsen, Czech Republic; \\ ${ }^{4}$ Biomedical Center, Faculty of Medicine in Pilsen, Charles University, Pilsen, Czech Republic
}

\begin{abstract}
Aim: The aim of this study was to evaluate the ability of tissue polypeptide-specific antigen (TPS), carcinoembryonic antigen (CEA), and cancer antigen 15-3 (CA 15-3) to predict relapse in breast cancer patients, when the measurement of biomarkers is performed within 6 months after surgery. Patients and Methods: Four hundred and seventy-two patients with breast cancer were evaluated.TPS, CEA, and CA 15-3 were measured in months 1, 3, and 6, after surgery. Disease recurrence was recorded between 7 12 months after surgery. Disease recurrence occurred in 60 patients, while 412 patients remained in recurrence-free status. Results: TPS levels of the recurrence group differed statistically significantly in the first and sixth month after surgery compared to recurrence-free group $(p=0.0339$, $A U C=0.6056 ; p<0.0001, A U C=0.7196) . C E A$ and $C A 15-3$ measurements did not achieve a statistically significant difference for any month examined. Conclusion: TPS level in the sixth month after surgery is the best candidate biomarker to predict disease recurrence.
\end{abstract}

Breast cancer is the most common malignancy and the second most frequent cause of death by cancer in women (breast cancer represents $24 \%$ of all cancers occurring in women). Although the incidence rate of breast cancer has been increasing, the mortality rate has remained stable for decades.

Correspondence to: Asst. Prof. Radek Kucera, Department of Immunochemistry, University Hospital Pilsen, Edvarda Benese 13, 30599 Pilsen, Czech Republic. Tel: +420 377402948, Fax: +420 377402454, e-mail: kucerar@fnplzen.cz

Key Words: Breast cancer, surgery, monitoring, TPS, CEA, CA15-3.
This is particularly due to the earlier diagnosis and the effective screening programs as well as advances in treatment (1).

The efficacy of breast cancer treatment has gradually increased over recent years. Though, an improved follow-up management would enable a more effective therapy, particularly for patients with a high risk of tumor relapse (2). In other words, it would be useful to have a reliable, easily measurable prognostic factor. Tumor markers comprise a valuable tool that can be used for this purpose. Serum tumor markers are routinely used in diagnostics, therapy monitoring, and follow-up of patients with breast cancer. The predictive value of tumor markers has long been discussed (3). Several models have been tested starting with preoperative levels followed by preoperative and postoperative level comparisons and then, further routine measurement for an extended period of observation with evaluation of changes in tumor marker dynamics (4). In our study, we decided to perform the clinical measurements during a 6-month period after surgery. During this time patients are in close and frequent contact with the hospital due to postoperative examinations or adjuvant therapies.

The aim of this study was to evaluate the ability of carcinoembryonic antigen (CEA), cancer antigen 15-3 (CA 15-3), and tissue polypeptide specific antigen (TPS) to predict relapse in patients after radical resection of the tumor when the measurement of tumor markers is performed intensively during the first six months after surgery.

\section{Patients and Methods}

Group of patients. The study group included 472 patients who underwent radical surgery for breast cancer. The baseline patient characteristics are summarized in Table I. All the cancer diagnoses were histologically verified. The study was approved by the local ethical committee and all the patients signed an informed consent 
before participating in this study. Serum levels of TPS, CEA, and CA 15-3 were determined at the following intervals: 1, 3, and 6 months after surgery. After the third measurement of tumor markers we continued monitoring patients from the 7 th to the 12th month after surgery, and recorded the disease status. Based on these observations we divided the patients in two groups. The first group consisted of $60(13 \%)$ patients with disease recurrence (local recurrence or metastatic spread) and median age 61 years (minimum 32, maximum 88 ). The second group consisted of $412(87 \%)$ patients with no disease recurrence and median age 61 years (minimum 37, maximum $84)$. We compared the results of these two groups.

Serum samples. Samples of peripheral venous blood were collected using the VACUETTE blood collection system (Greiner Bio-one Company, Kremsmünster, Austria). Serum was separated by 10 minute centrifugation at $1300 \times g$, and immediately frozen to $-80^{\circ} \mathrm{C}$. Serum samples were thawed only once, just prior to analyses. According to the guidelines of the European Group on Tumor Markers (EGTM), the tumor markers were measured before chemotherapy or radiotherapy. Hormonal therapy administration was not a contraindication to tumor marker measurement (5).

Biomarker measurement. Serum levels of TPS were measured using the radioisotope IRMA TPS assay (IDL Biotech, Bromma, Sweden). CEA and CA 15-3 serum levels were measured using the chemiluminescent ACCESS CEA and ACCESS CA 15-3 assay (Beckman Coulter, Brea, CA, USA).

Statistical analysis. SAS 9.2 (Statistical Analysis Software release 9.2; SAS Institute Inc., Carry, NC, USA) was used for all statistical analyses. Receiver operating characteristic (ROC) curves were plotted and the area under the curve (AUC) was calculated. The Wilcoxon test was used to compare distributions of values between the groups of patients and $p$-value $<0.05$ indicated the statistical significance.

\section{Results}

We compared the relapsed and relapse-free groups. TPS showed statistically significant differences when measured in the first and sixth month after surgery. Median levels of TPS at the first month, were $75 \mathrm{IU} / 1$ and $45 \mathrm{IU} / 1(p=0.0339$, AUC=0.6056), respectively, and at the sixth month were $83 \mathrm{IU} / 1$ and $47 \mathrm{IU} / 1$ $(p<0.0001$, AUC $=0.7196)$, respectively (Table II). CEA showed statistically significant difference in the first month after surgery. Median levels of CEA at the first month, were $1.30 \mu \mathrm{g} / \mathrm{l}$ and $1.20 \mu \mathrm{g} / 1(p=0.0432, \mathrm{AUC}=0.5520)$, respectively (Table III). CA 15-3 showed statistically significant differences in the third month after surgery. Median levels of CA $15-3$ at the third month, were $14.0 \mathrm{kIU} / \mathrm{l}$ and $12.50 \mathrm{kIU} / \mathrm{l} \quad(p=0.0359$, AUC $=0.5590$ ), respectively (Table IV). According to the AUC values, TPS occupied the first two places followed by CA 15 3 , and then CEA. Figure 1 shows the four best ROC curves.

\section{Discussion}

In our university hospital, breast cancer patients are monitored for 6 months after radical surgery. After adjuvant chemotherapy or radiotherapy termination, the patient is usually transferred
Table I. Basic characteristics of patients.

\begin{tabular}{lc}
\hline Patients & 472 \\
Age (median) & 61 \\
Histology & $409(88.7 \%)$ \\
Ductal carcinoma & $24(5.1 \%)$ \\
Lobular carcinoma & $39(8.2 \%)$ \\
Other & \\
Stage & $220(46.6 \%)$ \\
IA & $132(28.0 \%)$ \\
IIA & $67(14.2 \%)$ \\
IIB & $38(8.1 \%)$ \\
IIIA & $9(1.9 \%)$ \\
IIIB & $6(1.3 \%)$ \\
IIIC & \\
Operation & $168(35.6 \%)$ \\
Mastectomy & $304(64.4 \%)$ \\
Partial resection & \\
Adjuvant therapy & $227(48 \%)$ \\
Adjuvant chemotherapy & $429(91 \%)$ \\
Adjuvant radiotherapy & $360(76 \%)$ \\
Adjuvant hormonal therapy & \\
Mitotic activity (MIB1) & $20(1-100)$ \\
Median (Min. - Max.) & \\
Hormonal receptors & $373(79 \%)$ \\
Estrogen-positive & $99(21 \%)$ \\
Estrogen-negative & $368(78 \%)$ \\
Progesterone-positive & $104(22 \%)$ \\
Progesterone-negative & \\
HER2/neu & $184(39 \%)$ \\
Positive & $288(61 \%)$ \\
Negative & \\
\hline
\end{tabular}

MIB1, E3 ubiquitin protein ligase 1; HER2, human epidermal growth factor receptor 2 .

to another hospital where follow-up is continued or monitoring continues at longer intervals. Therefore, we wanted to verify whether intensive tumor marker measurement and evaluation can be used as a prognostic factor to identify patients with a high risk of disease relapse in order to continue or recommend more intensive monitoring in these patients over the coming period. Earlier relapse detection also contributes to a lower mortality rate for this cancer disease (6).

CEA is one of the first recognized tumor markers. It is tumor-specific and not organ-specific (7). CA 15-3 detects soluble forms of mucin-1 (MUC-1) transmembrane oncoprotein, which is overexpressed in breast cancer. CA 153 , in combination with CEA, is the marker most used in breast cancer detection. CA 15-3 correlates with tumor mass, nodal status, and presence of metastatic disease (8). Multiplication of cytokeratins is one of the signs of cell division. During the progression of malignant tumors, there is observed a proteolytic cleavage of soluble cytokeratin fragments and their subsequent release into the blood (9). TPS is the marker of proliferative activity, while it also correlates with tumor size, stage, and grade (10). 
Svobodova et al: CEA, CA 15-3 and TPS in Monitoring of Breast Cancer Patients

Table II. Comparison of recurrence and recurrence-free groups, concerning tissue polypeptide specific antigen (TPS).

\begin{tabular}{|c|c|c|c|c|c|c|}
\hline TPS (IU/l) & Recurrence & Median & Minimum & Maximum & $p$-Value Wilcoxon test & AUC \\
\hline \multirow[t]{2}{*}{1 month } & YES & 75.0 & 17.0 & 1508 & $p=0.0271$ & 0.6056 \\
\hline & NO & 45.0 & 10.0 & 683 & & \\
\hline \multirow[t]{2}{*}{3 months } & YES & 52.0 & 10.0 & 507 & $p=0.1800$ & 0.5245 \\
\hline & NO & 51.5 & 10.0 & 438 & & \\
\hline \multirow[t]{2}{*}{6 months } & YES & 83.0 & 10.0 & 1065 & $p<0.0001$ & 0.7196 \\
\hline & NO & 47.0 & 6.50 & 906 & & \\
\hline
\end{tabular}

Data in bold indicate statistical significance. AUC, Area under the curve.

Table III. Comparison of recurrence and recurrence-free groups, concerning carcinoembryonic antigen (CEA).

\begin{tabular}{lcccccc}
\hline CEA $(\mu \mathrm{g} / \mathrm{l})$ & Recurrence & Median & Minimum & Maximum & $p$-Value (Wilcoxon test) & AUC \\
\hline \multirow{2}{*}{1 month } & YES & 1.30 & 0.45 & 41.9 & $\boldsymbol{p = 0 . 0 4 3 2}$ & 0.5520 \\
& NO & 1.20 & 0.42 & 8.00 & $p=0.1342$ & 0.5257 \\
3 months & YES & 1.40 & 0.50 & 23.9 & & \\
& NO & 1.30 & 0.10 & 5.20 & 0.0713 & 0.5345 \\
6 months & YES & 1.40 & 0.50 & 6.00 & & \\
& NO & 1.20 & 0.30 & 5.10 & & \\
\hline
\end{tabular}

Data in bold indicate statistical significance. AUC, Area under the curve.

Table IV. Comparison of recurrence and recurrence-free groups, concerning cancer antigen 15-3 (CA 15-3).

\begin{tabular}{|c|c|c|c|c|c|c|}
\hline CA 15-3 (kIU/l) & Recurrence & Median & Minimum & Maximum & $p$-Value Wilcoxon test & AUC \\
\hline \multirow[t]{2}{*}{1 month } & YES & 11.6 & 10.0 & 25.0 & \multirow[t]{2}{*}{$p=0.2512$} & \multirow[t]{2}{*}{0.5363} \\
\hline & NO & 11.0 & 4.60 & 15.6 & & \\
\hline \multirow[t]{2}{*}{3 months } & YES & 14.0 & 7.50 & 70.0 & \multirow[t]{2}{*}{$p=0.0359$} & \multirow[t]{2}{*}{0.5590} \\
\hline & NO & 12.5 & 5.20 & 35.3 & & \\
\hline \multirow[t]{2}{*}{6 months } & YES & 13.0 & 6.10 & 33.0 & \multirow[t]{2}{*}{$p=0.1063$} & \multirow[t]{2}{*}{0.536} \\
\hline & NO & 12.0 & 4.70 & 34.0 & & \\
\hline
\end{tabular}

Data in bold indicate statistical significance. AUC, Area under the curve.

The first studies regarding predictive values of CEA, CA 15-3, and TPS in breast cancer recurrence were conducted during the 80s and 90s. Given et al. reported CA 15-3, followed by TPS, as the most sensitive individual tumor marker to predict breast cancer recurrence. CEA was found to be less useful for this purpose. Better results were achieved by a combination of CA 15-3 and TPS (11). Further studies were mainly focused on the role of CA 15-3. Authors tried to establish the cut-off value of CA 15-3 at the time of first recurrence, which could serve as a predictive factor. The cut-off was established at $30 \mathrm{kIU} / 1$ and $47 \mathrm{kIU} / \mathrm{l}$, respectively $(12,13)$. The turn of the millennium saw the focus turn towards the intensive use of tumor markers and several authors tried to apply new procedures in treatment indication. Studies conducted by Nicolini and coworkers can serve as an example. In particular, these studies included an innovative procedure, based on 3 tumor markers, CA 15-3, CEA, and tissue polypeptide antigen (TPA), for the indication of salvage treatment. There were 2 patient groups, the first was treated just after tumor marker level elevation, while the second after the confirmation of metastasis using imaging techniques, which corresponded to the usual guidelines. Their results revealed significantly higher survival of the first group, indicating tumor marker-guided treatment as more successful than imaging-based treatment. However, guidelines for cancer treatment indication have not been changed yet $(14,15)$. Other authors have reported the ability of CA 15-3 and CEA to be good predictors of distant 


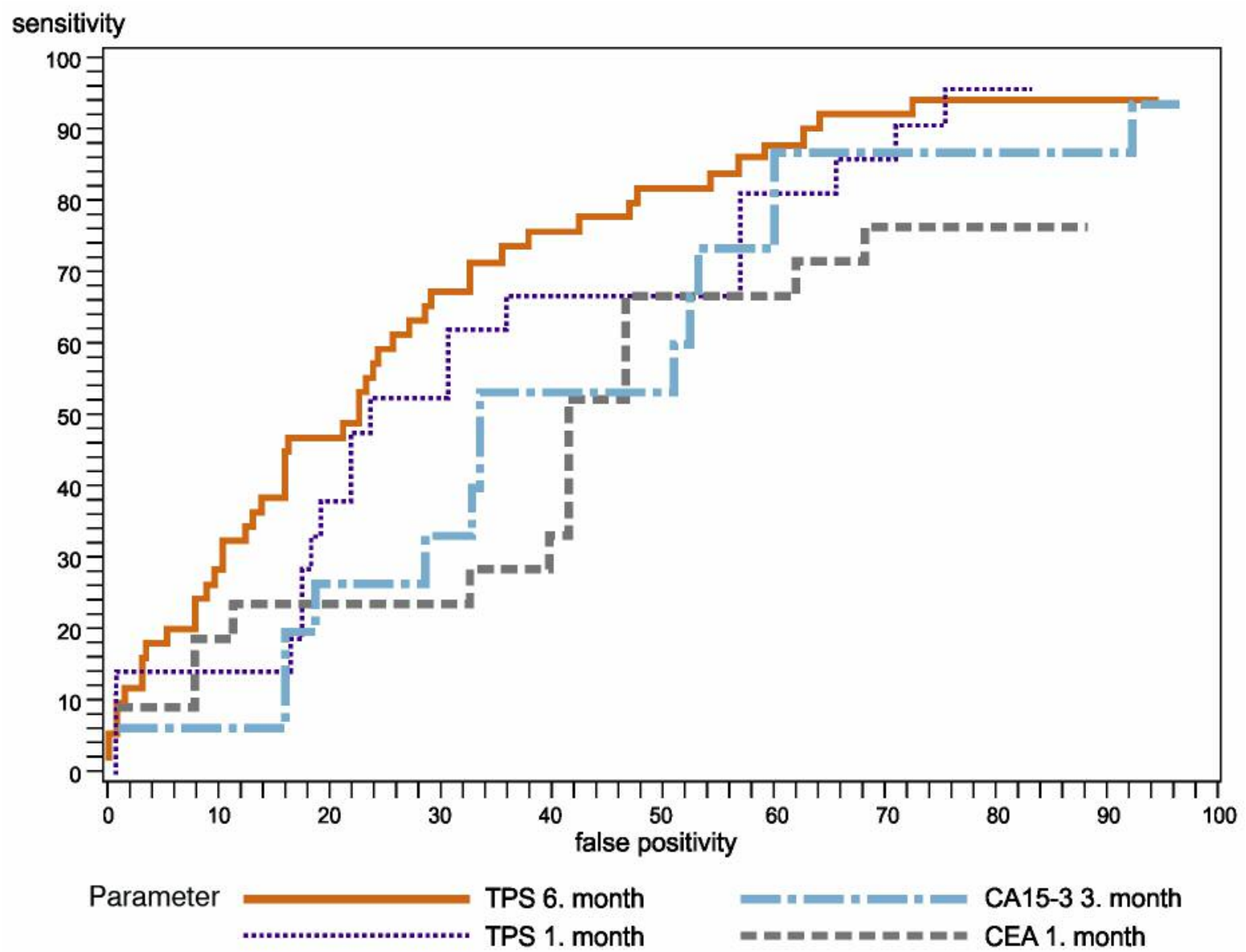

Figure 1. ROC analyses. The four best ROC curves for breast cancer recurrence. ROC, Receiver operating characteristic; TPS, tissue polypeptidespecific antigen; CA 15-3, cancer antigen 15-3; CEA, carcinoembryonic antigen.

metastases, though poor predictors of local recurrence (16, 17). A recent study by Shao et al. confirmed preoperative serum levels of CEA and CA15-3 as independent prognostic parameters for breast cancer (18). During the last years, studies on breast cancer prognosis are focused on multivariate models with the use of blood tests for CEA, CA15-3, serum human epidermal growth factor receptor 2 (HER2), lactate dehydrogenase, C-reactive protein and alkaline phosphatase, combined with further parameters like estrogen receptor positivity, metastatic status and other clinical parameters $(19,20)$.

Based on the findings of the above mentioned studies and our own experience, we have designed a postoperative surveillance algorithm and further confirmed it in clinical practice.

The present study indicated that TPS is a better prognostic marker of breast cancer relapse than CEA and CA 15-3. TPS was the best marker (according to the AUC values) measured in months 1 and 6 after surgery. Hence, our results suggest that TPS should be added to the panel of biomarkers for the follow-up monitoring of breast cancer patients, after surgery. The optimal timing for sample collection for biomarker testing is the sixth month after surgery, when the adjuvant chemotherapies and radiotherapies are terminated.

In conclusion, TPS was evaluated as the best candidate for the prediction of breast cancer recurrence, since it achieved the highest AUC in the sixth month after surgery.

\section{Acknowledgements}

This study was supported by Ministry of Health, Czech Republic conceptual development of research organization Faculty Hospital in Pilsen - FNPl, 00669806 and by the project CZ.1.05/2.1.00/03.0076 from European Regional Development Fund. 


\section{References}

1 Rojas K and Stuckey A: Breast cancer epidemiology and risk factors. Clin Obstet Gynecol 59: 651-672, 2016.

2 Benjamin DJ: The efficacy of surgical treatment of cancer - 20 years later. Med Hypotheses 82: 412-420, 2014.

3 Duffy MJ: Serum tumor markers in breast cancer: are they of clinical value? Clin Chem 52: 345-351, 2006.

4 Duffy MJ, O'Donovan N, McDermott E and Crown J: Validated biomarkers: The key to precision treatment in patients with breast cancer. Breast 29: 192-201, 2016.

5 Molina R, Barak V, van Dalen A, Duffy MJ, Einarsson R, Gion M, Goike H, Lamerz R, Nap M, Sölétormos G and Stieber P: Tumor markers in breast cancer- European Group on Tumor Markers recommendations. Tumor Biol 26: 281-293, 2005.

6 Vilaprinyo E, Puig T and Rue M: Contribution of early detection and adjuvant treatments to breast cancer mortality reduction in Catalonia, Spain. PLoS One 1: e30157, 2012.

7 Yasasever V, Dincer M, Camlica H, Karaloglu D and Dalay N: Utility of CA 15-3 and CEA in monitoring breast cancer patients with bony metastases: special emphasis on 'spiking' phenomena. Clin Biochem 30: 53-56, 1997.

8 Park BW, Oh JW, Kim JH, Park SH, Kim KS, Kim JH and Lee KS: Preoperative CA 15-3 and CEA serum levels as predictor for breast cancer outcomes. Ann Oncol 19: 675-681, 2008.

9 Barak V, Frenkel S, Valyi-Nagy K, Leach L, Apushkin MA, Lin AY, Kalickman I, Baumann NA, Pe'er J, Maniotis AJ and Folberg R: Using the direct-injection model of early uveal melanoma hepatic metastasis to identify TPS as a potentially useful serum biomarker. Invest Ophthalmol Vis Sci 48: 43994402, 2007.

10 O'Hanlon DM, Kerin MJ, O'Boyle C, Grimes H and Given HF: Tissue polypeptide specific antigen (TPS) in breast cancer - an initial evaluation. Eur J Surg Oncol 22: 38-41, 1996.

11 Given M, Scott M, Mc Grath JP and Given HF: The predictive of tumor markers CA 15-3, TPS and CEA in breast cancer recurrence. Breast 9: 277-280, 2000.

12 Tampellini M, Berruti A, Gerbino A, Buniva T, Torta M, Gorzegno G, Faggiuolo R, Cannone R, Farris A, Destefanis M, Moro G, Deltetto F and Dogliotti L: Relationship between CA 15-3 serum levels and disease extent in predicting overall survival of breast cancer patients with newly diagnosed metastatic disease. Br J Cancer 75: 698-702, 1997.
13 De La Lande B, Hacene K, Floiras JL, Alatrakchi N and Pichon MF: Prognostic value of CA 15.3 kinetics for metastatic breast cancer. Int J Biol Markers 17: 231-238, 2002.

14 Nicolini A, Anselmi L, Michelassi C and Carpi A: Prolonged survival by 'early' salvage treatment of breast cancer patients: a retrospective 6-year study. Br J Cancer 76: 1106-1111, 1997.

15 Nicolini A, Carpi A, Michelassi C, Spinelli C, Conte M, Miccoli P, Fini $\mathrm{M}$ and Giardino R: "Tumor marker guided" salvage treatment prolongs survival of breast cancer patients: final report of a 7-year study. Biomed Pharmacother 57: 452-459, 2003.

16 Mariani L, Miceli R, Michilin S and Gion M: Serial determination of CEA and CA 15.3 in breast cancer follow-up: an assessment of their diagnostic accuracy for the detection of tumor recurrences. Biomarkers 14: 130-136, 2009.

17 Lumachi F, Basso SM, Bonamini M, Marzano B, Milan E, Waclaw BU and Chiara GB: Relationship between preoperative serum markers CA 15-3 and CEA and relapse of the disease in elderly ( $>65$ years) women with breast cancer. Anticancer Res 30: 2331-2334, 2010.

18 Shao Y, Sun X, He Y, Liu C and Liu H: Elevated levels of serum tumor markers CEA and CA15-3 are prognostic parameters for different molecular subtypes of breast cancer. PLoS One 24: e0133830, 2015.

19 Di Gioia D, Dresse M, Mayr D, Nagel D, Heinemann V and Stieber P: Serum HER2 in combination with CA 15-3 as a parameter for prognosis in patients with early breast cancer. Clin Chim Acta 440: 16-22, 2015.

20 Nieder C, Dalhaug A, Haukland E, Mannsaker B and Pawinski A: Prognostic impact of the tumor marker CA $15-3$ in patients with breast cancer and bone metastases treated with palliative radiotherapy. J Clin Med Res 9: 183-187, 2017.

Received October 9, 2017

Revised October 20, 2017

Accepted October 26, 2017 